\title{
GRISOTTO: A greedy approach to improve combinatorial algorithms for motif discovery with prior knowledge
}

\author{
Alexandra M Carvalho ${ }^{1^{*}}$ and Arlindo L Oliveira ${ }^{2}$
}

\begin{abstract}
Background: Position-specific priors (PSP) have been used with success to boost EM and Gibbs sampler-based motif discovery algorithms. PSP information has been computed from different sources, including orthologous conservation, DNA duplex stability, and nucleosome positioning. The use of prior information has not yet been used in the context of combinatorial algorithms. Moreover, priors have been used only independently, and the gain of combining priors from different sources has not yet been studied.

Results: We extend RISOTTO, a combinatorial algorithm for motif discovery, by post-processing its output with a greedy procedure that uses prior information. PSP's from different sources are combined into a scoring criterion that guides the greedy search procedure. The resulting method, called GRISOTTO, was evaluated over 156 yeast TF ChIP-chip sequence-sets commonly used to benchmark prior-based motif discovery algorithms. Results show that GRISOTTO is at least as accurate as other twelve state-of-the-art approaches for the same task, even without combining priors. Furthermore, by considering combined priors, GRISOTTO is considerably more accurate than the state-of-the-art approaches for the same task. We also show that PSP's improve GRISOTTO ability to retrieve motifs from mouse ChiP-seq data, indicating that the proposed algorithm can be applied to data from a different technology and for a higher eukaryote.

Conclusions: The conclusions of this work are twofold. First, post-processing the output of combinatorial algorithms by incorporating prior information leads to a very efficient and effective motif discovery method. Second, combining priors from different sources is even more beneficial than considering them separately.
\end{abstract}

\section{Background}

An important part of gene regulation is mediated by specific proteins, called transcription factors (TF), which influence the transcription of a particular gene by binding to specific sites on DNA sequences, called transcription factor binding sites (TFBS). Such binding sites are relatively short segments of DNA, normally 5 to 25 nucleotides long. Discovering TFBS's is a challenging task, mainly because they exhibit a high degree of degeneracy making them difficult to distinguish from random artifacts. For this reason, algorithms for motifs discovery often suffer from impractical high false positive rates and return noisy models that are not useful to

\footnotetext{
* Correspondence: asmc@kdbio.inesc-id.pt

'Department of Electrical Engineering, IST/TULisbon, KDBIO/INESC-ID, Lisboa, Portugal

Full list of author information is available at the end of the article
}

characterize TFBS's. Some extra knowledge, carefully selected from the literature, has been incorporated in motif discovery methods in order capture a variety of characteristics of the motif patterns. This extra knowledge is used during the process of motif discovery.

Some interesting works in this line of research made use of the DNA structure for motif discovery. These works take into consideration the bendability of a region, as well as the nucleotide position in DNA loops, to determine sequence accessibility [1-3]. A quite different and particularly interesting work was devised by R. Lavery [4-10]. In one approach [4], the atomic structure of the protein, which specifically bounds to a fragment of DNA, was used to calculate the binding energy needed for the full combinatorial space of base sequences. Binding sites were selected considering an energy cutoff. This result suggests that the crystallographic structure of a protein-DNA

\section{Ciomed Central}


complex indeed contains enough information to locate the binding sequences of a protein. Recently, a general approach was proposed which allows the incorporation of almost any type of information into the class of motif discovery algorithms based on Gibbs sampling [11]. This extra information is incorporated in a position-specific prior (PSP) and it amounts for the likelihood that a motif starts in a certain position of a given DNA sequence. The most effective PSP's have been built in a discriminative way by taking into account not only the sequence-sets that were bounded by some profile TF, but also sequence-sets that were not bounded. This is accordant to the evidence that the discovery of regulatory elements is improved by employing discriminative approaches [12]. A PSP is built in pre-processing time and then used to bias the optimization procedure towards real motifs. Prior information such as orthologous conservation, DNA duplex stability, nucleosome positioning and transcription factor structural class have been shown to be very effective when used with Gibbs sampler-based PRIORITY algorithm [11,13-16]. The popular MEME algorithm also pointed out that PSP's are beneficial when used with EM procedures [17]. This approach has not yet been used in the context of combinatorial algorithms for the same task. Moreover, the information given by PSP's from different sources was never combined, although there is evidence that predicting protein-DNA interactions can be improved by integrating diverse information [18].

Meanwhile, chromatin immunoprecipitation (ChiP) followed by ultra-high-throughput sequencing, known as ChiP-seq, brought new challenges for motif discovery [19]. As a result of direct sequencing of all DNA fragments from ChiP assays, ChiP-seq is able to unravel DNA sites, across the entire genome, where a specific protein binds. Regions of high sequencing read density are referred to as peaks to capture the evidence of high base-specific read coverage. Peaks are found by peak finding algorithms [20], which is called peak calling, yielding a set of DNA fragments of ChiP-enriched genomic regions. Usually, DNA fragments of size $\pm 100 \mathrm{bp}$ are extracted around top peaks and then a motif discovery tool is used to find for overrepresented sequences [21]. Some authors have further exploited the information provided by these binding peaks by devising priors that use coverage profiles as motif positional preferences [22,23].

In this paper, we extend the RISOTTO combinatorial algorithm [24] in a greedy fashion to take into account prior information in a PSP format. RISOTTO is a consensus-based algorithm that exhaustively enumerates all motifs of a certain size by collecting their occurrences, at a given distance, from a set of co-regulated DNA sequences [24-27]. Since methods based on the detection of overrepresentation of TFBS's in co-regulated
DNA sequences are known to face problems detecting weak motifs, we propose to post-process the RISOTTO output by modifying top motifs in a greedy fashion, guided by the information given by the prior. The rational for this approach is that the combinatorial algorithm exploits the full space of possible motifs pointing out good candidates. Afterwards a greedy search is performed over these initial guesses and good motifs are up-weighted by the prior. The reduction of the search space attained in the greedy search by using the output of a combinatorial algorithm makes the proposed algorithm, called GRISOTTO, very efficient.

A great advantage of GRISOTTO is its ability to combine priors from different sources. This is achieved by considering a convex combination of the information given by all priors resulting in an information-theoretical scoring criterion called Balanced Information Score (BIS). To unravel the benefits of using BIS with GRISOTTO we focus on discovering motifs in 156 benchmark datasets from ChIP-chip data from yeast. We considered three different priors already used by PRIORITY, namely, orthologous conservation $[14,16]$, DNA duplex stability [15] and nucleosome positioning [11]. By combining the information of these three priors together in BIS we guided the GRISOTTO greedy search and achieved considerably more accurate results than by using the priors separately. Moreover, we further verified that GRISOTTO is at least as accurate as the PRIORITY and MEME algorithms when using the same priors separately.

We also gauge GRISOTTO with 13 mouse ChiP-seq data. In this evaluation we used two different priors providing extra information from orthologous conservation [17] and coverage profiles given by ChiP-seq assays [23]. Results show that orthologous conservation was able to uncover motifs that resemble ones already reported by previous works on the same data $[17,21]$. However, the PSP built from the ChiP-seq assays was not very beneficial to GRISOTTO, as it reported exactly the same motifs as the uniform prior for which any position in the DNA sequences is likely to contain a motif. We attributed this to the fact that the information contained in this prior is already encoded in the BIS score. Indeed, coverage profiles indicate overrepresentation, expressed via high sequencing read density, and the BIS score is a weighted balance between overrepresentation and priors.

Besides effectiveness, GRISOTTO also showed to be very efficient, taking around 2 to 3 seconds per yeast sequence-set, that have around 200 sequences of $500 \mathrm{bp}$, and 1 to 4 minutes per mouse sequence-set, that have from around 1000 to 40000 sequences of 200 bp. These computational times were obtained using one core of an Intel $2.4 \mathrm{GHz}$ Core 2 Duo and include the generation of the initial starting points by RISOTTO. 
We conclude that post-processing the output of combinatorial algorithms guided with the information given by single or combined priors yields an efficient approach that shows great promise in extending the power of motif discovery tools.

\section{Methods}

Herein, we present the GRISOTTO algorithm for motif discovery. The proposed algorithm uses the RISOTTO [24] output as starting points of a greedy procedure that aims at maximizing a scoring criterion based on combined prior information. Our approach diverges from EM (used in MEME [17]) and Gibbs sampling (used in PRIORITY [11,13-16]) as we do not consider latent variables and do not use a background model. Moreover, instead of maximizing the likelihood, we propose a scoring criterion based on the balanced information of observing the DNA sequences and the priors given a candidate motif. We called this score Balanced Information Score (BIS). Furthermore, instead of reporting a PSSM, GRISOTTO returns the IUPAC string that is best fitted, according to BIS, via a greedy search procedure.

\section{GRISOTTO algorithm}

We next introduce some notation needed to describe the GRISOTTO algorithm (refer to Table 1). Start by considering that we have a set of $N$ co-regulated DNA sequences henceforward denoted by $f=\left(f_{i}\right)_{i}=1, \ldots, N$. The length of the each sequence $f_{i}$ is $n_{i}$, that is, $f_{i}=\left(f_{i j}\right)_{j=1}, \ldots, n_{i}$. Moreover, consider that $S_{p}$ contains some prior information in a PSP format about the domain in study, with $p=1 \ldots \ell$, where $\ell$ is the number of priors (eventually zero). We denote by $S=\left\langle S_{1}, \ldots, S_{\ell}\right\rangle$ the list of all priors. The goal of GRISOTTO is to report a single motif of a fixed size $k$, that is, an IUPAC string of size $k$. The IUPAC alphabet is henceforward denoted by $\Sigma$.

The pseudocode of GRISOTTO is depicted in Algorithm 1 . The algorithm starts by running RISOTTO to extract, at least $z_{\text {min }}$, and at most $z_{\text {max }}$, motifs of size $k$ (see details in Additional File 1). From the RISOTTO output, the top $z$ motifs are collected in a set called $\mathcal{C}$ (Step 2) and constitute the starting points of the GRISOTTO greedy procedure, called GGP (Step 4). Briefly, GGP starts with a motif $m \in \mathcal{C}$ and returns the best fitted motif, according to BIS, by updating each position in $m$ with an IUPAC symbol until no local improvements can be achieved. In Step 5-6 the variable $r$, that stores the output of the algorithm, is updated whenever the GGP procedure returns a motif with a BIS score higher than the current stored one. Note that in Step 2 the result variable $r$ is initialized with the empty motif $\varepsilon$. We consider that the empty motif $\varepsilon$ has the minimum possible BIS scoring value.

Table 1 Definition of terms used in describing the algorithms presented in Methods.

\begin{tabular}{|c|c|}
\hline Symbol & Meaning \\
\hline$\Sigma$ & alphabet (usually DNA or IUPAC) \\
\hline$f$ & input sequences \\
\hline$f_{i}$ & $i$-th input sequence \\
\hline$f_{i j}$ & $j$-th position of the $i$-th input sequence \\
\hline N & number of input sequences \\
\hline$n_{i}$ & length of $f_{i}$ \\
\hline k & motif size \\
\hline$S_{p}$ & $p$-th prior (in PSP format) \\
\hline$\ell$ & number of priors (it can be zero) \\
\hline$S$ & $S=\left\langle S_{1}, \ldots, S_{l}\right\rangle$ is the list of all priors \\
\hline$z_{\min }$ & minimum number of motifs expected to be returned by a RISOTTO run \\
\hline$z_{\max }$ & maximum number of motifs expected to be returned by a RISOTTO run \\
\hline$z$ & number of top motifs post-processed from RISOTTO output \\
\hline $\mathcal{C}$ & the set with the $z$ top motifs to be post-processed by GRISOTTO \\
\hline$m$ & motif of size $k$ \\
\hline$m\langle i, \alpha\rangle$ & motif $m$ where the $i$-th position (starting with 0 ) is replaced by $\alpha \in \Sigma$ \\
\hline$\varepsilon$ & empty motif (its BIS score is the minimum possible value) \\
\hline$f_{i}[j \ldots j+k-1]$ & $k$-long segment of the $i$-th input sequence that starts at position $j$ \\
\hline$S_{p}[i, j]$ & prior probability at the $j$-th position of $f_{i}$ \\
\hline$j_{i}$ & annotated position for $f_{i}$ with maximum BIS score for a motif $m$ \\
\hline$P_{m}$ & probability distribution given by the PSSM induced by $m$ \\
\hline$\alpha_{p}$ & the weight of the $p$-th prior \\
\hline$\lambda$ & coefficient to balance priors and over-representation contribution \\
\hline
\end{tabular}




\section{Algorithm 1 GRISOTTO, Greedy RISOTTO}

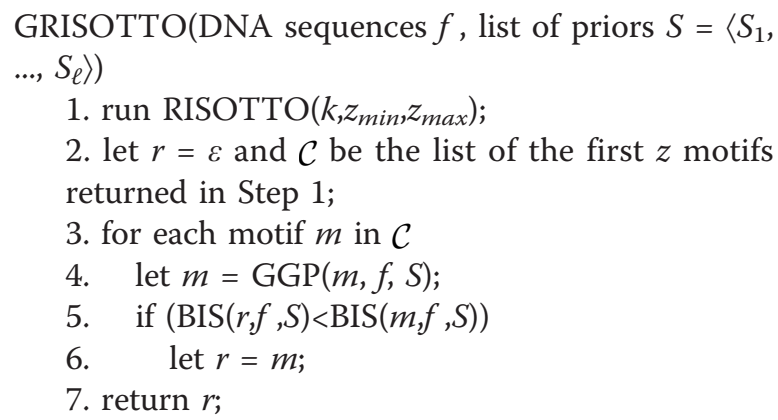

It remains to explain the GGP procedure given in Algorithm 2. The general idea of the algorithm is to process each position of the motif $m$, received as parameter, in a greedy fashion. Variable $i$ identifies the motif position being processed. It is initialized with the value 0 (Step 1), the first position of $m$, and it is incremented in a circular way using modular arithmetics (Step 9). GPP terminates when $k$ consecutive positions of the motif $m$ being considered can not be improved, according to BIS, and so $m$ remains unchanged for a complete $k$-round. This information is stored in variable $t$ that counts how many consecutive positions of $m$ have not been modified. Variable $t$ is initialized with 0 (Step 1) and controls the outer cycle (Step 2-9), which terminates when $t=k$. The Boolean flag changed is read in the outer cycle (Step 7) to detect whether the $i$-th position of the motif has been modified inside the body of the inner cycle (Step 6). It is initialized in each run of the outer cycle with false (Step 3). The inner cycle (Step 4-6) tries to improve the BIS score of $m$ by updating its $i$-th position with each letter $\alpha \in \Sigma$. We denote by $m\langle i$, $\alpha>$ the motif $m$ where the $i$-th position of $m$ was replaced by the letter $\alpha$. Whenever the BIS score of $m\langle i$, $\alpha\rangle$ is greater than the BIS score of $m$ (Step 5) three variables are updated: (i) motif $m$ is updated to $m\langle i, \alpha\rangle$; (ii) variable $t$ is reset to its initial value, forcing a complete $k$-round from that point on; and (iii) flag changed is turned to true. After the inner cycle, in Step 7, we test whether the $i$-th position of $m$ was not modified by checking the value of the flag changed. If that is the case, variable $t$ is incremented (Step 8). Next, in Step 9, variable $i$ is incremented so that the next position of $m$ can be inspected.

Algorithm 2 GGP, GRISOTTO greedy procedure

GGP(motif $m$, DNA sequences $f$, list of priors $S=$ $\left.\left\langle S_{1}, \ldots, S_{\ell}\right\rangle\right)$
1. let $t=0$ and $i=0$;
2. while $(t<k)$
3. let changed $=$ false;
4. for each $\alpha$ in $\Sigma$

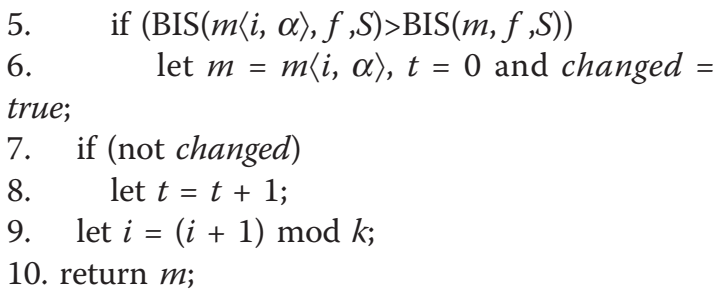

We note that the GGP procedure converges since the BIS score is upper-bounded. Next, we derive and present in detail the BIS score.

\section{Balanced information score}

Start by noticing that a motif $m$ of size $k$ written in IUPAC can be easily translated into a PSSM with dimension $4 \times k$ (for details see Additional file 1). Moreover, observe that if we had to guess in which position $m$ occurs in sequence $f_{i}$ that would be the position $j_{i}$ that maximizes $P_{m}\left(f_{i}\left[j_{i} \ldots j_{i}+k-1\right]\right)$ where $\mathrm{P}_{\mathrm{m}}(w)$ is the probability of observing the DNA word $w$ by the PSSM induced by $m$ and $f_{i}\left[j_{i} \ldots j_{i}+k-1\right]$ is the $k$-long segment of $f_{i}$ that starts at position $j_{i}$. In other words, such $j_{i}$ annotates the position in which we believe the motif $m$ occurs in $f_{i}$. Henceforward consider that we annotate for each sequence $f_{i}$ the respective position $j_{i}$ where $m$ occurs with higher probability (refer to Table 1).

Following Shannon, the self-information of a probabilistic event with probability $p$ is given by $-\log (p)$. If the event is very rare, the self-information is very high. On the other hand, if the event has probability close to 1 , observing such event gives us almost no information. So, by assuming that $m$ occurs independently in each sequence of $f$, the self-information that $m$ occurs in all sequences of $f$ in the annotated positions is given by

$$
\sum_{i=1}^{N}-\log \left(P_{m}\left(f_{i}\left[j_{i} \ldots j_{i}+k-1\right]\right)\right) .
$$

Note that the above sum is zero (its minimal value) if the motif $m$ occurs with probability 1 in all annotated positions and, moreover, the sum is not upper-bounded.

Considering that the priors are in PSP format, their information can be easily computed from the annotated sequences. Indeed, the self-information given by the prior $S_{p}$ of observing the annotated positions $j_{i}$, for all $1 \leq i \leq N$, is computed as

$$
\sum_{i=1}^{N}-\log \left(S_{p}\left[i, j_{i}\right]\right),
$$

where $S_{p}[i, j]$ is the prior probability stored at the $j$-th position of the $i$-th sequence in the $S_{p}$ PSP file. Having this, it remains to understand how the information from different priors can be combined. Actually, priors come 
from different sources [11,13-16], and some of these sources might have more quality or be more relevant for motif discovery than others. A simple way to heuristically combine prior information is to multiply the contribution of each prior by a constant $\alpha_{p}$ that measures the belief in the quality/relevance of each prior $S_{p}$ and consider a balanced sum of all self-informations. In order to keep the resulting value with the same magnitude of each component, we consider a convex combination, that is, $\sum_{p=1}^{\ell} \alpha_{p}=1$. Thus, the combined self-information is computed as

$$
\sum_{p=1}^{\ell}\left(\alpha_{p} \sum_{i=1}^{N}-\log \left(S_{p}\left[i, j_{i}\right]\right)\right) .
$$

Following a similar idea, we balance with a constant $\lambda$ $\in(0,1]$ the self-information given by the occurrence of the motif in (1) with the self-information given by the priors in (2), obtaining in this way the following expression:

$$
\begin{aligned}
\lambda \sum_{i=1}^{N}- & \log \left(P_{m}\left(f_{i}\left[j_{i} \ldots j_{i}+k-1\right]\right)\right)+(1-\lambda) \sum_{p=1}^{\ell}\left(\alpha_{p} \sum_{i=1}^{N}-\log \left(S_{p}\left[i, j_{i}\right]\right)\right)= \\
& -\sum_{i=1}^{N}\left(\lambda \log \left(P_{m}\left(f_{i}\left[j_{i} \ldots j_{i}+k-1\right]\right)+(1-\lambda) \sum_{p=1}^{\ell} \alpha_{p} \log \left(S_{p}\left[i, j_{i}\right]\right)\right)\right) .
\end{aligned}
$$

The closer the above expression is to zero the less (balanced) self-information follows from observing a candidate motif $m$ in the annotated positions of both the DNA sequences and the priors. Indeed, we expect motifs to occur in the annotated positions of both the DNA sequences and the priors with high probability. Therefore, the goal is to find a motif $m$ that minimizes such information. Next, and for the sake of simplification, we drop the minus sign in (3), that is, we consider the final scoring criterion, called balanced information score (BIS), defined as

$$
\operatorname{BIS}(m, f, S)=\sum_{i=1}^{N}\left(\lambda \log \left(P_{m}\left(f_{i}\left[j_{i} \ldots j_{i}+k-1\right]\right)+(1-\lambda) \sum_{p=1}^{\ell} \alpha_{p} \log \left(S_{p}\left[i, j_{i}\right]\right)\right),\right.
$$

and restate our goal to finding a motif $m$ that maximizes (4). Note that $\operatorname{BIS}(m, f, S)$ is always non-positive and, therefore, is upper-bounded by 0 .

For the BIS score in Equation (4) to be well-defined it remains to determine the values of the constants $\lambda$ and $\alpha_{p}$ for all $1 \leq p \leq \ell$. Whenever there is no knowledge about the quality of the priors the values of such constants should be uniform, that is, $\lambda=\frac{1}{2}$ and $\alpha_{p}=\frac{1}{\ell}$ for all $1 \leq p \leq \ell$. Usually, it is possible to refine heuristically these constants by evaluating the usefulness of each prior in well-know domains.

Finally, it is not obvious how to translate back the combined information into a combined prior that could be used in an EM or Gibbs sampler-based algorithm. These techniques need that such prior reflects the probability of finding a motif in a certain position of the DNA sequences in order to correctly bias, in each iteration step, the expected log-likelihood of the candidate motif occurring in the positions given by the latent variable. On the other hand, GRISOTTO incorporates prior information in BIS resulting in a theoretical-information scoring criterion that measures the information of observing the candidate motif in the annotated positions of both the DNA sequences and the priors. These annotated positions are computed only once, for each candidate motif, in such a way that the balanced contribution to the BIS score of the DNA sequences and the priors in those positions is maximal. The higher the value of the BIS score, the higher the probability that a candidate motif occurs in the annotated positions of both the DNA sequences and the priors. Therefore, GRISOTTO reports the motif, among all candidate ones, that maximizes the BIS scoring criterion.

\section{Results}

The GRISOTTO algorithm was implemented in Java. Source code and binaries are available at http://kdbio. inesc-id.pt/ asmc/software/grisotto.html. A C implementation of the RISOTTO combinatorial algorithm, needed by GRISOTTO, is also available. Source code and executables can also be found at the GRISOTTO webpage.

We start the evaluation of the effectiveness of GRISOTTO by measuring the benefits of using single and combined priors in finding motifs in yeast ChiP-chip data. This data is now a gold standard with several priors available, providing an unbiased experimental assay for motif discovery tools. It contains a humancurated set of 156 motifs known to be present in 156 sequence-sets (one motif per sequence-set). Motif finder tools are asked to report a single motif for each sequence-set, which is then compared with the humancurated one. Human-curated motifs are called throughout this work as literature motifs, known motifs or even true motifs. Details about the data, priors, evaluation methodology, and results can be found in the following ChiP-chip data subsection.

We also provide an additional check on the value of using priors with GRISOTTO from data with different characteristics - a higher eukaryote with sequence data derived from a different technology. On this account, we evaluate the performance of GRISOTTO in 13 sequence-sets from mouse ChiP-seq data. Details of this assessment can be found in ChiP-seq data subsection.

\section{ChiP-chip data}

We gauge the performance of GRISOTTO by measuring the benefits of using BIS for finding motifs in 
156 sequence-sets experimentally verified to bind different TF's in yeast. These datasets were collected by PRIORITY researchers [11] and were compiled from the work of Harbison et al. [28]. More precisely, Harbison et al. profiled the intergenetic binding locations of 203 TF's under various environmental conditions over 6140 yeast intergetecic regions. From these only intergenetic sequences reported to be bounded with a $p$-value $\leq$ 0.001 for some condition were considered by the PRIORITY researchers. Moreover, only sequence-sets with at least size 10 bounded by TF's with a known consensus from the literature were considered, resulting in 156 sequence-sets. Presently, these datasets are being used to benchmark several motif discovery tools [11,14-17,28-35] as they provide a reliable and fair assay over real data.

Three different PSP's were incorporated in BIS to boost GRISOTTO motif discoverer, namely, priors based on evolutionary conservation [14,16], destabilization energy [15], and nucleosome occupancy [11]. All these priors were devised by PRIORITY researchers and were kindly made available by the authors (personal communication). The popular MEME algorithm was also evaluated with the evolutionary conservation-based prior [17] devised by PRIORITY researchers. Since the sequence-sets and priors used to evaluate GRISOTTO were exactly the ones used in PRIORITY and MEME and, moreover, the criterion used to determine a correct prediction by the algorithms was also the same, we were able to make direct comparisons with their published results. PRIORITY and MEME had already shown that the use of these priors is advantageous when combined with Gibbs sampling and EM techniques. Herein we aim at investigating if the same improvements are also achieved by GRISOTTO. Moreover, we evaluate if combining priors is beneficial.

Following the approach of PRIORITY, we let GRISOTTO look for a single motif of size 8 in each of the 156 yeast sequence-sets, since priors were computed for 8-mers. The results provided by MEME considered a modification of the priors, adapting them for $k$-mers of different sizes. As a consequence, MEME was able to report accurately a large number of long motifs. Although we acknowledge that MEME's approach improves the capacity to discover motifs, we keep the original priors used in PRIORITY. Moreover, to measure the accuracy of GRISOTTO we used exactly the same metric as the one previously used by the PRIORITY and MEME researches. This metric compares the single motif reported by the discoverer, for each of the 156 yeast sequence-sets, to a literature motif by computing a scaled version of the Euclidean distance between the true motif and the reported one. A more complete explanation of this metric can be found in Additional file 1.
The results of GRISOTTO, as well as those of stateof-the-art motif discoverers, are summarized in Table 2. Detailed results of GRISOTTO can be found in Additional file 2 while details about the evaluation methodology, including, parameter settings and running times, can be found in Additional file 1. A brief explanation about the priors is given in the following sections.

\section{Evolutionary conservation-based priors}

Diverse methods for motif discovery make use of orthologous conservation to assess wether a particular DNA site is conserved across related organisms, and thus more likely to be functional. A comprehensive work along this line was done by PRIORITY researchers $[14,16]$, where an orthologous conservation-based prior was devised to improve their Gibbs sampler-based motif discovery method. This prior was built in a discriminative way by taking into account not only sequence-sets that were bounded by some profiled TF (the positive set) but also sequence-sets that were not bounded by the same TF (the negative set). In this way the prior reflects not only the probability that a $W$-mer at a certain position is conserved but of all the conserved occurrences of this $W$-mer what fraction occurs in the bound sequence-set. Conserved occurrences are found by searching if a $W$-mer in a reference sequence also occurs in most of its orthologous ones regardless of its orientation or specific position. For this particular case, the evolutionary conservation-based prior was used for each intergenetic region in $S$. cerevisiae and it used the orthologous sequences from six related organisms, namely, S. paradoxus, S. mikatae, S. kudriavzevii, S. bayanus, S. castelli and S. kluyveri. The prior was named discriminative conservation-based prior $(\mathcal{D C})$ and was made available, in a PSP format, at PRIORITY webpage.

Herein, we gauge the performance of GRISOTTO when this exact $\mathcal{D C}$ prior is incorporated into the BIS scoring criterion. Results comparing GRISOTTO-DC with PRIORITY-DCC[16], MEME- $\mathcal{D C}[17]$, and other state-of-the-art algorithms, can be found in Table 2 . Results show that GRISOTTO-DC correctly predicted 83 motifs out of the 156 experiments, whereas PRIORITY-DC found 77 and MEME:ZOOP-DC 81 . We conclude that GRISOTTO performed at least as well as PRIORITY and MEME:ZOOP when the same $\mathcal{D C}$ PSP was used. A closer inspection of detailed results of GRISOTTO, in Additional file 2 reveals that GRISOTTO$\mathcal{D C}$ found 15 motifs that PRIORITY-DC did not, while PRIORITY-DC found only 10 motifs that GRISOTTO$\mathcal{D C}$ did not.

\section{Destabilization energy-based priors}

Information about DNA double-helical stability has also been collected into a PSP to boost the PRIORITY Gibbs sampler-based algorithm [15]. The rational 
Table 2 Comparison of GRISOTTO with state-of-the-art methods over ChiP-chip data.

\begin{tabular}{|c|c|c|c|c|}
\hline Algorithm & Description & Successes & $\%$ & Ref \\
\hline PhyloCon & Local alignment of conserved regions & 19 & $12 \%$ & [29] \\
\hline PhyME & Alignment-based with EM & 21 & $13 \%$ & [30] \\
\hline MEME:OOPS & MEME with OOPS model & 36 & $23 \%$ & [31] \\
\hline MEME:ZOOPS & MEME with ZOOPS model & 39 & $25 \%$ & [31] \\
\hline MEME-C & MEME without conserved bases masked & 49 & $31 \%$ & [28] \\
\hline PhyloGibbs & Alignment-based with Gibbs Sampling & 54 & $35 \%$ & [32] \\
\hline Kellis et al. & Alignment-based & 56 & $36 \%$ & [33] \\
\hline CompareProspector & Alignment-based with Gibbs sampling & 64 & $41 \%$ & [34] \\
\hline Converge & Alignment-based with EM & 68 & $44 \%$ & [35] \\
\hline MEME:OOPS-DC & MEME with OOPS model and $\mathcal{D C}$ priors & 73 & $47 \%$ & {$[17]$} \\
\hline PRIORITY- $\mathcal{D C}$ & Gibbs sampler with $\mathcal{D C}$ priors & 77 & $49 \%$ & [16] \\
\hline MEME:ZOOP- $\mathcal{D C}$ & MEME with ZOOPS model and $\mathcal{D C}$ priors & 81 & $52 \%$ & [17] \\
\hline GRISOTTO- $\mathcal{D C}$ & GRISOTTO with $\mathcal{D C}$ priors & 83 & $53 \%$ & - \\
\hline 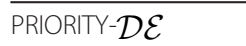 & Gibbs sampler with $\mathcal{D} \mathcal{E}$ priors & 70 & $45 \%$ & {$[15]$} \\
\hline GRISOTTO- $\mathcal{D} \mathcal{E}$ & GRISOTTO with $\mathcal{D} \mathcal{E}$ priors & 80 & $51 \%$ & - \\
\hline 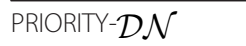 & Gibbs sampler with $\mathcal{D} \mathcal{N}$ priors & 70 & $45 \%$ & {$[11]$} \\
\hline GRISOTTO- $\mathcal{D} \mathcal{N}$ & GRISOTTO with $\mathcal{D} \mathcal{N}$ priors & 77 & $49 \%$ & - \\
\hline$\overline{\text { GRISOTTO-CDP }}$ & GRISOTTO with combined priors & 93 & $60 \%$ & - \\
\hline
\end{tabular}

The results of motif discoverers were taken from R. Gordân et al. [16] and T. L. Bailey et al. [17].

All priors used were devised by R. Gordân, A. J. Hartemink and L. Narlikar [11,14-16].

for the information contained in this prior is based in the fact that, in general, the energy needed to destabilize the DNA double helix is higher at TFBS's than at random DNA sites. The PSP resulting from this effort was built in a discriminative way, just as for the $\mathcal{D C}$ prior, and was named discriminative energy-based prior $(\mathcal{D E})$

We evaluated the $\mathcal{D E}$ prior within GRISOTTO.

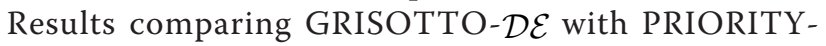
$\mathcal{D E}[15]$, and other state-of-the-art algorithms, can be

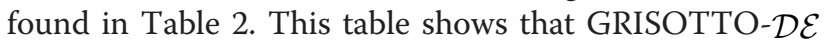
correctly predicted 80 motifs out of the 156 experiments, whereas PRIORITY-DEE found only 70 . We conclude that GRISOTTO performed quite well when the $\mathcal{D E}$ prior was used, with an improvement of $14 \%$ over correct predictions relatively to PRIORITY, raising the overall proportion of successful predictions in 6\% (from $45 \%$ to $51 \%$ ). As before, we made a closer examination of the detailed results included in Additional file 2

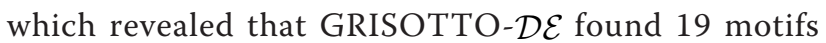

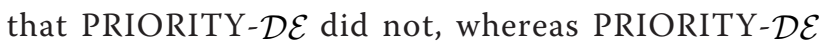
found only 9 motifs that GRISOTTO-DEE did not.

\section{Nucleosome occupancy-based priors}

Nucleosome occupancy has also been used in motif discovery. The rationale for this approach is that Eukaryotic genomes are packaged into nucleosomes along chromatin affecting sequence accessibility. There are two main works in the literature to predict genomewide organization of nucleosomes in Saccharomyces cerevisiae [36-38]. Taking into account the work of Segal et al. [38] the PRIORITY researchers [11] devised an informative prior based on a discriminative view of nucleosome occupancy. The prior was named discriminative nucleosome-based prior $(\mathcal{D N})$.

GRISOTTO was evaluated with the $\mathcal{D} \mathcal{N}$ prior incorporated in the BIS score. Results comparing GRISOTTO-D $\mathcal{N}$ with PRIORITY-DN $\mathcal{N}$, and other state-ofthe-art algorithms, can be found in Table 2. This table shows that GRISOTTO-DN $\mathcal{N}$ correctly predicted 77 motifs out of the 156 experiments, while PRIORITY-DC found 70. We conclude that GRISOTTO outperformed PRIORITY when $\mathcal{D} \mathcal{N}$ prior was used, with an improvement of $10 \%$ over correct predictions. A closer investigation of detailed results in Additional file 2 unravels that GRISOTTO- $\mathcal{D} \mathcal{N}$ found 13 motifs that PRIORITY$\mathcal{D N}$ did not, whereas PRIORITY-DN $\mathcal{N}$ found 6 motifs that GRISOTTO-DN $\operatorname{did}$ not.

\section{Combining priors}

Despite considerable effort to date in developing new potential priors to boost motif discoverers, PSP's from different sources have not yet been combined. Actually, although having some degree of redundancy, because, for instance, the positioning of nucleosomes may be correlated with DNA double helix stability, it is easy to conclude by a closer inspection of the detailed results in Additional file 2 that different PSP's still report a considerable number of disjoint motifs (refer to Additional file 1 for further details). As a matter of fact, PRIORITY researchers have already noticed this fact [15]. However, it is not a trivial task determining how to translate the 
BIS combined information into a PSP that can be used in EM or Gibbs sampler-based algorithms.

In order to gauge the potential of combined priors, we incorporated in the BIS score the three $\mathcal{D C}, \mathcal{D E}$ and $\mathcal{D N}$ priors. We call the final prior combined discriminative prior $(\mathcal{C D} \mathcal{P})$. Results show that GRISOTTO-CDP is the more accurate motif discoverer for the 156 sequence-sets being evaluated. It correctly predicted 93 motifs, while GRISOTTO-DC found 83, GRISOTTO$\mathcal{D E} 80$ and GRISOTTO-D $\mathcal{N}$ 77. In this way GRISOTTO-CDP accomplished an improvement of at least $12 \%$ over correct predictions, when compared with GRISOTTO variants considering the priors individually. This raises the overall proportion of successful predictions in $7 \%$, on top of the improvements already attained in the previous sections, over these 156 yeast sequence-sets. Moreover, when comparing GRISOTTO$\mathcal{C D} \mathcal{P}$ with state-of-the-art motif discoverers (refer to Table 2), the final proportion of successful predictions was raised to $60 \%$, while the best known previous value, to our knowledge, was $51 \%$ attained by MEME- $\mathcal{D C}[17]$. This leads us to conclude that combining priors from different sources is even more beneficial than considering them separately.

\section{ChiP-seq data}

Herein we measure the accuracy of GRISOTTO in TF motif discovery on 13 mouse ChiP-seq data. This data was gathered by Chen et al. [21] where whole-genome binding sites of 13 sequence-specific TFs (Nanog, Oct4, STAT3, Smad1, Sox2, Zfx, c-Myc, n-Myc, Klf4, Essrb, Tcfcp2l, E2f1, and CTCF) were profiled in mouse ES cells using the ChiP-seq approach. Sequences of \pm 100 bp size from the top 500 binding peaks were selected for each factor, repeats were masked, and the Weeder [39] tool was used to find overrepresented sequences unravelling 12 of the 13 factors (excluding E2f1).

We assess the quality of GRISOTTO in discovering motifs from mouse ChiP-seq data with two priors. First, an orthologous conservation-based PSP was used as information for higher organisms is now available. Indeed, there are already such PSP's for yeast, fly, mouse and even human $[14,16,17]$. Second, a binding peak-based PSP was tried as ChiP-seq assays provide an intrinsic positional prior that can be computed from base-specific coverage profiles. This prior has recently been employed in motif discoverers [22,23] with success.

As for ChiP-chip data, we let GRISOTTO find for a single motif of size 8 , since priors were computed for 8 mers. However, as human-curated motifs are not available for this ChiP-seq data, we made only a resemblance, based on a 6-window match, between the motifs reported by GRISOTTO with those outputted by Chen et al. [21] and MEME [17] for the same data.

\section{Evolutionary conservation-based priors}

Orthologous conservation-based priors for mouse ChiPseq data were obtained by MEME researchers [17] following a similar methodology as PRIORITY-DC for the yeast ChiP-chip data ones. As before, this new mouse prior received the shorthand name $\mathcal{D C}$. We incorporated the $\mathcal{D C}$ prior into the BIS score and ran GRISOTTO. In Figure 1, motifs reported by Chen et al. and MEME- DC are shown along side motifs found by GRISOTTO-DC for the 13 mouse sequence-sets. Recall that Chen et al. only reported 12 out of the 13 motifs, excluding the E2f1 motif, so in this case the TRANSFAC [40] motif is shown instead. MEME-DC and GRISOTTO-DC were able to retrieve all motifs. Moreover, the number of sequences of these sequence-sets vary from 1038 to 38238 and, due to efficiency issues, MEME- $\mathcal{D C}$ was only able to run over 100 sequences randomly chosen from each sequence-set. GRISOTTO-DC was able to use all of them taking only 1-4 minutes, per sequence-set, to report a motif.

Because sequences-sets are very large, some of the reported motifs became highly degenerated. Actually, only 6 out of the 13 motifs seem to be highly conserved, namely, CTCF, Esrrb, Klf4, n-Myc, Tcfc and c-Myc. For these, even allowing for IUPAC symbols during the greedy search results in highly conserved motifs. Therefore, for this data, we searched for IUPAC strings that allow only two positions to have degenerate IUPAC symbols.

By a closer inspection of Figure 1 we conclude that motifs reported by GRISOTTO-DC are strongly similar to the ones reported by Chen et al. and MEME- $\mathcal{D C}$. Have in mind that GRISOTTO outputs an IUPAC, and not a PSSM, but we used, in a 6-window size, the same color scheme as PSSM's to make the resemblance with reported motifs easier.

\section{Binding peak-based priors}

$\mathrm{Hu}$ el al. [23] devised a prior using coverage profile information provided by the ChiP-seq approach. This grounds in the belief that motifs are tightly packed near the peak summit - the location inside each peak with the highest sequence coverage depth. As a result, prior probabilities were set to be proportional to a discretized Student's $t$-distribution with 3 degrees of freedom and rescaled such that they form a step function with a fixed 25 bp step-size. The prior probabilities are symmetric and centered at the peak summits. As such prior is intrinsically a positional one we built a PSP resuming the described probabilities for the 13 mouse ChiP-seq data and ran GRISOTTO.

Our results show that direct use of binding peak-based priors does not help GRISOTTO much. Actually, the motifs reported by this prior were exactly the same as using the uniform prior (recall that for the uniform 
Carvalho and Oliveira Algorithms for Molecular Biology 2011, 6:13

Page 9 of 13 http://www.almob.org/content/6/1/13
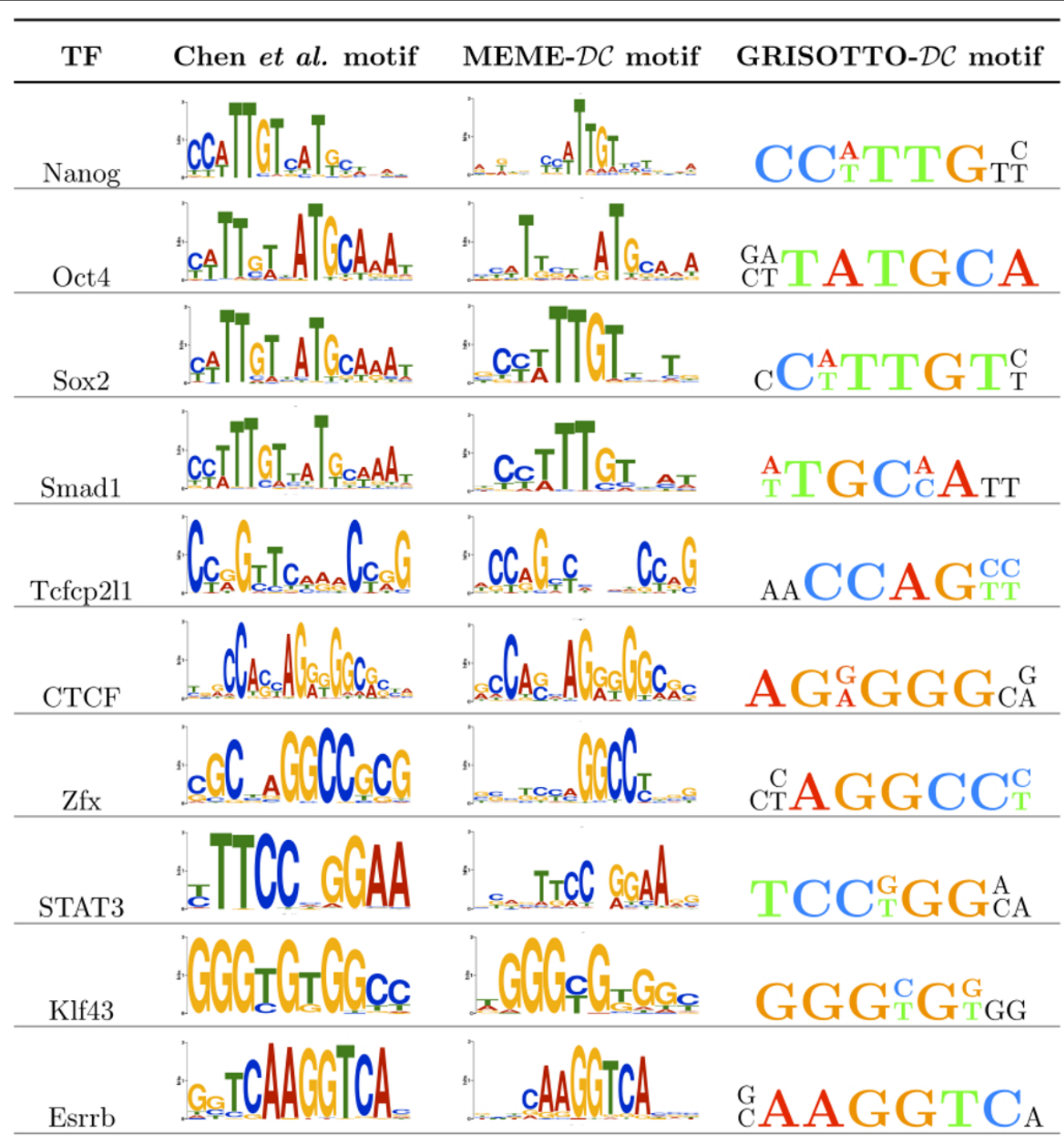

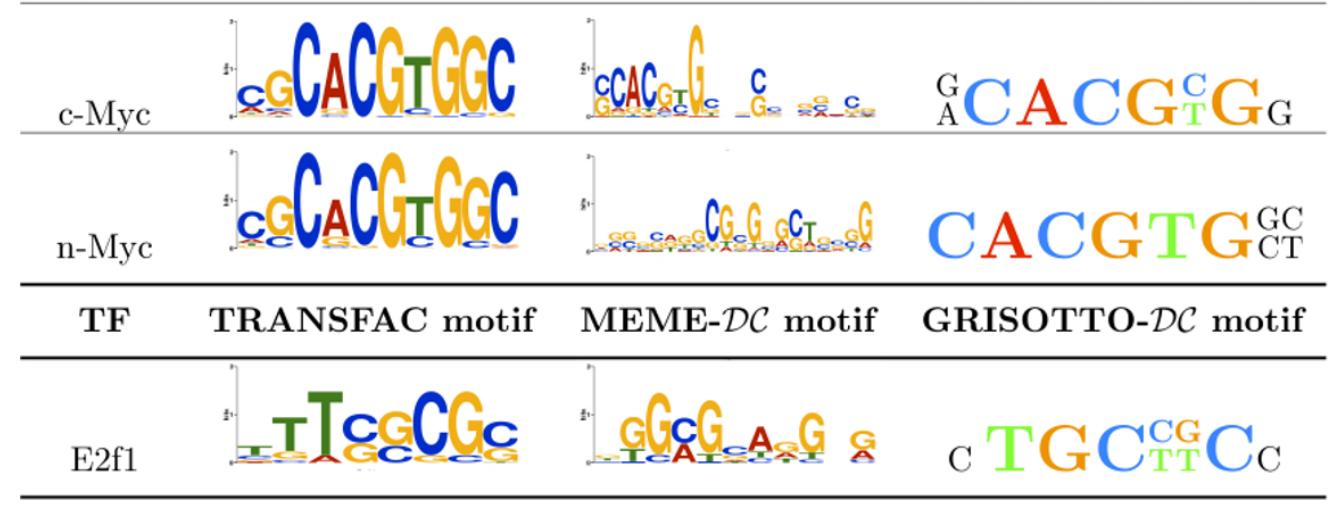

Figure 1 Comparison of GRISOTTO- $\mathcal{D C}$ with Chen et al. and MEME- $\mathcal{D C}$. Motifs reported by Chen et al. [21] and MEME- $\mathcal{D C}[17]$ are shown

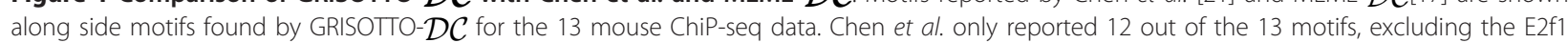
motif, so in this case the TRANSFAC [40] motif is shown instead. 
prior any position in the DNA is likely to contain a motif). Moreover, when combined with the $\mathcal{D C}$ prior GRISOTTO reported precisely the same motifs as $\mathcal{D C}$ prior alone. These findings suggest that GRISOTTO is unable to retrieve any useful information from the binding peak-based prior. We attributed this to the fact that part of the information contained in the binding peakbased prior is already encoded in the BIS score. Indeed, peak summits indicate an overrepresentation of a motif in a certain locus. Such overrepresentation is already weighted in the BIS score (recall Equation (1) and (4) in page 8-9). Notwithstanding, it seems reasonable that for short sequences of $200 \mathrm{bp}$ (namely, $\pm 100 \mathrm{bp}$ around the peak summits) the coverage-based prior has no real impact on motif discovery. For longer sequences, the effective resolution of the peak summits seems to provide useful information [22,23].

\section{Discussion}

Wasserman and Sandelin [41] noticed that the discovery of TFBS's from a nucleotide sequence alone suffers from impractical high false positive rates. This was termed the futility theorem as nearly every predicted TFBS has no function in vivo. This problem has been studied and addressed by taking into consideration information in and beyond the TFBS's, such as orthologous conservation $[16,17]$, nucleosome positioning [11,42], DNA duplex stability [14] and coverage profiles obtained from ChiP-seq assays [22,23].

Following this line of research we have verified in the present study that post-processing the output of RISOTTO with prior knowledge from different sources is beneficial for motif discovery. RISOTTO is a consensus-based method that enumerated exhaustively all motifs by collecting their occurrences, up to a fixed Hamming distance, from input sequences. The Hamming distance between two string measures the minimum number of substitutions required to change one string into the other. As a result, a set of overrepresented motifs is reported and then ordered by their biological relevance according to some statistical significance test $[24,26,27]$. This ordered list is retrieved in a classical way from the nucleotide sequence alone and, as previously mentioned, it is of particular importance to introduce a bias from available priors. Following this goal, we noticed that the top 10 motifs from the RISOTTO ordered list could be greedily modified to have a good BIS score. The greedy procedure would modify these motifs introducing some noise allowed by the prior and up-weighting weak motifs that were masked during the combinatorial and/or statistical significance test. Certainly, we would not expect RISOTTO, or any other combinatorial algorithm, to report completely outlandish motifs, as motif discovery problem is indeed a combinatorial problem that accounts for overrepresentation of a string in a set of DNA sequences. However, prior information provides valuable guidance on how to describe a motif that goes beyond neighborhoods (defined by the Hamming distance or any similar distance) of the consensus sequence. GRISOTTO incorporates such information in the BIS score providing in this way a broader definition of overrepresentation of a motif in the input sequences.

Currently, a significant point of discussion is related with the use of prior information as a post-processing step of RISOTTO, and not within the RISOTTO procedure itself. For the sake of simplicity, consider we are looking for motifs of a fixed size $k$. Combinatorial algorithms take into consideration overrepresentation of motifs to extract them. This extraction is exhaustive, by iteratively extending candidate strings of size $1 \ldots k-1$, letter by letter of the DNA alphabet, and checking in each step if the extended string is still overrepresented in the sequence-set. Usually, complex data structures, such as suffix-trees, are employed to extend the candidate string. Whenever an extension fails to be overrepresented in the input sequences that extension is disregarded and another one is attempted. Only extensions that reach the size $k$ are reported.

Conversely, prior information only asserts if a subsequence of a fixed size $\mathrm{W}$ in a certain position of the DNA sequences is likely to be a motif. It is not straightforward to use prior information in combinatorial algorithms because they would need to know if a sub-string of size $1 \ldots k-1$ is likely to be a motif. However, in one hand, it is space-wise unfeasible to have priors for multiple values of $W$. On the other hand, priors for small or large values of $W$ have no information whatsoever, as either they are very common (occur in all input sequences) or very rare (occur only once or never). Our work, as well as state-of-the-art ones [11,14-17], have shown that an efficient and effective solution is to consider $W=k=8$.

Besides this discussion, there are two obvious advantages of using prior information at a post-processing step. First, the greedy-search procedure is independent from the starting points provided by the combinatorial algorithm, allowing any method to be employed (for instance, Weeder [39], SMILE [26], RISO [27], RISOTTO, etc). Another advantage is that while new priors are devised, we do not need to re-compute previous starting points, being sufficient to run the greedy-search procedure of the GRISOTTO algorithm.

In closing, we stress that the BIS score was used throughout the experiments with sequence-sets known to be bound by a TF. Therefore, it was only used to discover the positions of each sequence-set where the motif occurs. Another possible application of the BIS 
score would be to detect the fraction of sequences that are likely to have site predictions. There are two possible ways to adapt GRISOTTO to this new problem: (i) derive a threshold of the BIS score contribution of a sequence above which the sequence is likely to have site predictions; (ii) incorporate an input parameter in the GRISOTTO greedy procedure, usually called quorum, that amounts for the fraction of sequences that have binding site predictions. None of these approaches seems straightforward and are out of the scope of this paper, hence they were left as a future research topic.

\section{Conclusions}

The GRISOTTO algorithm post-processes in a greedyfashion the output of RISOTTO taking into account prior information available about the domain. In practice, this introduces some extra knowledge taken from the literature, or computed from the sequences, that will help in characterizing motifs. The algorithm is flexible enough to combine several priors from different sources. Each prior is given a weight reflecting the confidence on the information contained in it and its relevance for motif discovery. In this way, priors can be introduced at will giving rise to a scoring criterion based on the convex closure of the information given by each prior.

Prior information has previously been shown to be beneficial when used with EM and Gibbs sampler-based motif discoverers. We have shown here that they can also be of great benefit to boost combinatorial algorithms such as RISOTTO. We emphasize that the goal of this paper is not to introduce new priors, but to show that priors can also be advantageous to assist and improve the output of combinatorial algorithms such as RISOTTO. Moreover, we have shown that combining priors is very promising in further extending the power of motif discovery algorithms.

We gauge the effect of adding prior information to GRISOTTO over 156 well-studied sequence-sets from yeast TF ChiP-chip experiments. For each sequenceset, motif discoverers were asked to report a single PSSM motif that was then compared with the known PSSM for the TF pulled down in the ChIP-chip experiment. Prior information from different sources was used, including, orthologous conservation, nucleosome occupancy, and destabilization energy. The use of exactly the same priors in EM and Gibbs samplerbased motif discoverers, namely, MEME and PRIORITY, respectively, has been shown to dramatically improve their performance. In this work, we show that this boost can be also achieved by GRISOTTO that performed at least as well as PRIORITY and MEME when each prior was considered individually. The great advantage of GRISOTTO was accomplished by the combination of priors. Indeed, when GRISOTTO compromised the three mentioned priors in a convex combination of their information it achieved an improvement of about $15 \%$ over correct predictions relatively to the best motif discoverer (MEME- $\mathcal{D C}[17])$, at our present knowledge, for exactly the same experiments. The final proportion of successful predictions is now at $60 \%$, attained with 93 correct predictions from GRISOTTO-CDP (with only 81 correct predictions of MEME-DC) out of the 156 experiments.

Finally, we also confirm the benefit of using GRISOTTO with 13 sequence-sets from a higher eukaryote ChiP-seq data, namely, the mouse. In this assessment two priors were used, including, orthologous conservation and base coverage profiles obtained from the ChiP-seq assays. We concluded that, as for ChiP-chip data, the orthologous conservation-based prior was of great convenience, being able to unravel 13 motifs strongly similar to the ones reported by other tools and found in the TRANSFAC database. In respect to the coverage-based prior, their direct use as a positional prior was not favorable, having been comparable to the uniform prior. We believe this is due to the fact that the BIS score already accounts for overrepresentation in the input sequences which we suspect mimics the information contained in this new prior, turning the prior redundant.

\section{Additional material}

Additional file 1: Detailed set up and evaluation methodology of
GRISOTTO. This additional file presents in detail three topics needed to
make the paper self-contained. First, is describes the call to RISOTTO
algorithm found in Step 1 of the Algorithm 1. Second, it includes the
inter-motif distance used to compute successful predictions from motif
discoverers, along with PSSM representation of IUPAC strings reported by
GRISOTTO. Finally, it contains relevant information about the evaluation
methodology, including, parameter settings and running times. This
makes the results presented in this paper reproducible along with the
data and algorithms provided in the GRISOTTO webpage.
Additional file 2: Detailed results of GRISOTTO. Additional details
about experimental results of GRISOTTO presenting actual predictions
sequence-set by sequence-set for various positional priors. It also
presents results of PRIORITY taken from the supplementary material of
the original papers.

\section{Acknowledgements and funding}

The authors thank Raluca Gordân and her co-authors for providing the sequence-sets and respective priors for the yeast experiments. We also thank Timothy Bailey and his co-authors for making available the mouse ChiP-seq data and respective priors used in the experiments. For last, but not least, the authors are very thankful for the invaluable comments of the anonymous referees.

This work was supported by FCT (INESC-ID multiannual funding) through the PIDDAC Program funds and by the projects PTDC/SAU-MII/100964/2008, PneumoSyS - A systems biology approach to the role of pneumococcal carbon metabolism in colonization and invasive disease, and PTDC/EIA/ 67722/2006, ARN - Algorithms for the Identification of Genetic Regulatory Networks, funded by FCT, Fundacão para a Ciência e Tecnologia. 


\section{Author details}

'Department of Electrical Engineering, IST/TULisbon, KDBIO/INESC-ID, Lisboa, Portugal. ${ }^{2}$ Department of Computer Science and Engineering, IST/TULisbon, KDBIO/INESC-ID, Lisboa, Portugal.

\section{Authors' contributions}

AMC did the programming and designed and performed the experiments. AMC also wrote the final draft of the paper. ALO did the proofreading of the final draft of the paper. Both authors have read and approved the final manuscript.

\section{Competing interests}

The authors declare that they have no competing interests.

Received: 10 November 2010 Accepted: 22 April 2011

Published: 22 April 2011

\section{References}

1. Beiko RG, Charlebois RL: GANN: Genetic Algorithm Neural Networks for the detection of conserved combinations of features in DNA. Proc. Natl Acad Sci USA 2005, 102:17400-17405.

2. Pudimat R, Schukat-Talamazzini EG, Backofen R: Feature Based Representation and Detection of Transcription Factor Binding Sites. Proc. German Conference on Bioinformatics 2004, 43-52.

3. Ponomarenko JV, Ponomarenko MP, Frolov AS, Vorobiev DG, Overton GC, Kolchanov NA: Conformational and physicochemical DNA features specific for transcription factor binding sites. Bioinformatics 1999, 15(7):654-668.

4. Deremble C, Lavery R: Macromolecular recognition. Current Opinion in Structural Biology 2005, 15:171-175.

5. O'Flanagan RA, Paillard G, Lavery $R$, Sengupta AM: Non-additivity in protein-DNA binding. Bioinformatics 2005, 21(10):2254-2263.

6. Paillard G, Lavery R: Analyzing protein-DNA recognition mechanisms. Structure 2004, 12:113-122.

7. Paillard G, Deremble C, Lavery R: Looking into DNA recognition: Zinc finger binding specificity. Nucleic Acids Research 2004, 32(22):6673-6682

8. Lafontaine I, Lavery R: ADAPT: A molecular mechanics approach for studying the structural properties of long DNA sequences. Biopolymers (Nucleic Acid Science) 2001, 56:292-310.

9. Lafontaine I, Lavery R: High-speed molecular mechanics searches for optimal DNA interaction sites. Comb Chem High Throughput Screen 2001, 4(8):707-717.

10. Lafontaine I, Lavery R: Optimization of nucleic acid sequences. Biophys J 2000, 79(2):680-685.

11. Narlikar L, Gordân R, Hartemink AJ: Nucleosome Occupancy Information Improves de novo Motif Discovery. Proc. RECOMB'07 2007, 107-121.

12. Valen E, Sandelin A, Winther O, Krogh A: Discovery of Regulatory Elements is Improved by a Discriminatory Approach. PLoS Comput Biol 2009, 5(11): e1000562.

13. Narlikar L, Gordân R, Ohler U, Hartemink AJ: Informative priors based on transcription factor structural class improve de novo motif discovery. Proc. ISMB'06 (Supplement of Bioinformatics) 2006, 384-392.

14. Gordân R, Narlikar L, Hartemink AJ: A Fast, Alignment-Free, ConservationBased Method for Transcription Factor Binding Site Discovery. Proc RECOMB'08 2008, 98-111.

15. Gordân R, Hartemink AJ: Using DNA Duplex Stability Information for Transcription Factor Binding Site Discovery. Pacific Symposium on Biocomputing 2008, 453-464.

16. Gordân R, Narlikar L, Hartemink AJ: Finding regulatory DNA motifs using alignment-free evolutionary conservation information. NuC AC Res 2010, 38(6):e90

17. Bailey $T L$, Bodén M, Whitington T, Machanick $P$ : The value of positionspecific priors in motif discovery using MEME. BMC Bioinformatics 2010, 11:179.

18. Ucar D, Beyer A, Parthasarathy S, Workman CT: Predicting functionality of protein-DNA interactions by integrating diverse evidence. 2009, 25(12): i137-i144.

19. Valouev A, Johnson DS, Sundquist A, Medina C, Anton E, Serafim Batzoglo and RMM, Sidow A: Genome-wide analysis of transcription factor binding sites based on ChIP-Seq data. Nature Methods 2008, 5:829-834.
20. Fejes AP, Robertson G, Bilenky M, Varhol R, Bainbridge M, Jones SJM: FindPeaks 3.1: a tool for identifying areas of enrichment from massively parallel short-read sequencing technology. Bioinformatics 2008, 24(15):1729-1730.

21. Chen $X, X u H$, Fang $F$ Pingand Yuan, Huss $M$, Vega VB, Wong $E$, Orlov $Y L$, Zhang W, Jiang J, Loh YH, Yeo HC, Yeo ZX, Narang V, Govindarajan KR, Leong B, Shahab A, Ruan Y, Bourque G, Sung WK, Clarke ND, Wei CL, $\mathrm{Ng} \mathrm{HH}$ : Integration of External Signaling Pathways with the Core Transcriptional Network in Embryonic Stem Cells. Cell 2008, 133(6):1106-1117.

22. Kulakovskiy IV, Boeva VA, Favorov AV, Makeev VJ: Deep and wide digging for binding motifs in ChIP-Seq data. Bioinformatics 2010, 26(20):2622-2623.

23. Hu M, Yu J, Taylor JM, Chinnaiyan AM, Qin ZS: On the detection and refinement of transcription factor binding sites using ChIP-Seq data. Nucleic Acids Research 2010, 38(7):2154-2167.

24. Pisanti N, Carvalho AM, Marsan L, Sagot MF: RISOTTO: Fast extraction of motifs with mismatches. In Proc. LATIN'06, Volume 3887 of LNCS. Edited by: JR Correa AH, Kiwi M. Spriger-Verlag; 2006:757-768.

25. Sagot MF: Spelling approximate repeated or common motifs using a suffix tree. Proc. Latin'98, Volume 1380 of LNCS. Edited by Lucchessi C, Moura A, Springer-Verlag 1998, 111-127.

26. Marsan L, Sagot MF: Algorithms for extracting structured motifs using a suffix tree with an application to promoter and regulatory site consensus identification. J Comp Bio 2000, 7(3-4):345-362.

27. Carvalho AM, Freitas AT, Oliveira AL, Sagot MF: An Efficient Algorithm for the Identification of Structured Motifs in DNA Promoter Sequences. IEEE/ ACM Trans. Comput Biol Bioinformatics 2006, 3(2):126-140.

28. Harbison CT, Gordon DB, Lee TI, Rinaldi NJ, Macisaac KD, Danford TW, Hannett NM, Tagne JB, Reynolds DB, Yoo J, Jennings EG, Zeitlinger J, Pokholok DK, Kellis M, Rolfe PA, Takusagawa KT, Lander ES, Gifford DK, Fraenkel E, Young RA: Transcriptional regulatory code of a eukaryotic genome. Nature 2009, 431(7004):99-104.

29. Wang T, Stormo GD: Combining phylogenetic data with co-regulated genes to identify regulatory motifs. Bioinformatics 2003, 19(18):2369-2380.

30. Sinha S, Blanchette M, Tompa M: PhyME: A probabilistic algorithm for finding motifs in sets of orthologous sequences. BMC Bioinformatics 2004, $5: 170$

31. Bailey $T L$, Elkan C: The value of prior knowledge in discovering motifs with MEME. Proc. ISMB'95 1995, 21-29.

32. Siddharthan R, Siggia ED, van Nimwegen E: PhyloGibbs: A Gibbs Sampling Motif Finder That Incorporates Phylogeny. PLoS Comput Biol 2005, 1(7): e67

33. Kellis M, Patterson N, Endrizzi M, Birren B, Lander ES: Sequencing and comparison of yeast species to identify genes and regulatory elements. Nature 2003, 423:241-254.

34. Liu Y, Liu S, Wei L, Altman RB, Batzoglou S: Eukaryotic Regulatory Element Conservation Analysis and Identification Using Comparative Genomics. Genome Res 2004, 14:451-458.

35. Maclsaac KD, Wang T, Gordon DB, Gifford DK, Stormo GD, Fraenkel E: An improved map of conserved regulatory sites for Saccharomyces cerevisiae. BMC Bioinformatics 2006, 7:113.

36. Lee C, Shibata Y, Rao B, Strahl B, Lieb J: Evidence for nucleosome depletion at active regulatory regions genome-wide. Nature Genetics 2004, 36(8).

37. Yuan GC, Liu YJ, Dion MF, Slack MD, Wu LF, Altschuler SJ, Rando OJ: Genome-scale identification of nucleosome positions in $S$ cerevisiae. Science 2005, 309(5734):626-630.

38. Segal E, Fondufe-Mittendorf $Y$, Chen L, Thåström A, Field $Y$, Moore IK, Wang JPZ, Widom J: A genomic code for nucleosome positioning. Nature 2006, 442(7104):772-778.

39. Pavesi G, Mereghetti P, Mauri G, Pesole G: Weeder Web: discovery of transcription factor binding sites in a set of sequences from coregulated genes. Nucleic Acids Research 2004, 32 Web-Server: 199-203.

40. Matys V, Kel-Margoulis OV, Fricke E, Liebich I, Land S, Barre-Dirrie A, Reuter I, Chekmenev D, Krull M, Hornischer K, Voss N, Stegmaier P, LewickiPotapov B, Saxel H, Kel AE, Wingender E: TRANSFACompel: transcriptional gene regulation in eukaryotes. Nucleic Acids Research 2006, , 34 Database: 108-110.

41. Wasserman WW, Sandelin A: Applied bioinformatics for the identification of regulatory elements. Nature reviews 2004, 5(4):276-287. 
42. Daenen F, van Roy F, Bleser PJD: Low nucleosome occupancy is encoded around functional human transcription factor binding sites. BMC Genomics 2008, 9(332).

doi:10.1186/1748-7188-6-13

Cite this article as: Carvalho and Oliveira: GRISOTTO: A greedy approach to improve combinatorial algorithms for motif discovery with prior knowledge. Algorithms for Molecular Biology 2011 6:13.

Submit your next manuscript to BioMed Central and take full advantage of:

- Convenient online submission

- Thorough peer review

- No space constraints or color figure charges

- Immediate publication on acceptance

- Inclusion in PubMed, CAS, Scopus and Google Scholar

- Research which is freely available for redistribution

Submit your manuscript at www.biomedcentral.com/submit
Ciomed Central 